\title{
On the intimate relationship between the principle of duality and the Hough transform
}

\author{
By Alberto S. Aguado ${ }^{1} \dagger$, Eugenia Montiel ${ }^{2} \dagger$ \\ AND MARK S. NIXON ${ }^{3}$ \\ ${ }^{1}$ INRIA Rhône-Alpes, ZIRST, 655 avenue de l'Europe, \\ 38330 Montbonnot Saint-Martin, France \\ ${ }^{2}$ iMAGIS/GRAVIR-IMAG, BP 35, 38041 Grenoble Cedex 9, France \\ ${ }^{3}$ Electronics and Computer Science, University of Southampton, \\ Southampton SO17 1BJ, UK
}

Received 8 July 1998; revised 19 April 1999; accepted 20 July 1999

This paper shows the relationship between the principle of duality and the Hough transform. We show that the definition of the Hough transform actually corresponds to an application of the principle of duality. In this interpretation, the general mathematical concepts of figures and high-dimensional coordinates in projective space are re-expressed in terms of shapes and mappings used for shape extraction in Euclidean space. So far, some works have demonstrated the equivalence between the definition of several concepts used in pattern matching and the Hough transform. These works have related the Hough transform to particular developments of diverse concepts including template matching, the Radon transform, maximum likelihood estimation and robust statistics. The correspondence between the Hough transform definition and the principle of duality here developed suggests a more general definition of the Hough transform with a deeper meaning in pure geometry. This definition introduces the formalism of projective geometry to shape extraction and analysis and thus the ideas, properties and geometric relationships in the projective space can have an interpretation for the development of pattern matching techniques. We discuss the analogy between the definition of a figure in geometry and the notion of a shape in the Hough transform, and how this relationship and the generalization of the principle of duality to high-dimensional spaces, defined by space coordinates, are related to the extensions of the Hough transform. Parametric forms are used to develop a dual analytic expression of general forms. The intimate relationship between the Hough transform and the principle of duality increases our understanding of the dual nature of pattern matching which can now benefit from established results in projective geometry.

Keywords: duality; Hough transform; computer vision;

shape extraction; projective geometry; pattern matching

$\dagger$ Present address: Department of Electronic and Electrical Engineering, University of Surrey, Guildford GU2 5XH, UK.

$\ddagger$ Present address: INRIA Rhône-Alpes, ZIRST, 655 avenue de l'Europe, 38330 Montbonnot SaintMartin, France. 


\section{Introduction}

In computer vision, perception has traditionally been recognized as a matching process wherein the establishment of corresponding geometric elements (i.e. pixels, points or curves) between patterns allows us to infer knowledge about a scene and to reason about objects (Julesz 1971; Marr \& Nishihara 1978; Pentland 1986). Thereby, the problem of pattern matching has been considered a central topic of research and has motivated the development of many techniques for segmentation, recognition and stereopsis, amongst others. Despite more than 30 years of research, this topic is still a fruitful field in computer vision and new algorithms, aimed at solving the fundamental problem of matching, continue to be developed.

In the extensive developments in pattern matching, different techniques have been proposed based on ideas from many scientific fields. Some approaches have been based on ideas from human vision, while others follow more applied approaches which focus on the quality of the results and computational properties of the algorithms. One technique that has played an important role in the development of pattern matching is the Hough transform (HT). This technique was originally developed to find bubble tracks rather than shapes in images (Hough 1962) and its potential as a coordinate conversion algorithm capable of line extraction was popularized by Rosenfeld (1969). Since then, the HT has been the subject of extensive research (Illingworth \& Kittler 1988; Leavers 1993) which has aimed primarily to increase its generality (the complexity of the shapes that can be extracted) while avoiding the large requirements of time and memory inherent in its implementation.

An important topic of research in the HT has focused on understanding and analysing the nature of its definition. In this aspect, some works have considered the relationship between the HT and other techniques. Perhaps the most relevant result in this area is the establishment of the equivalence between the HT and template matching (Stockman \& Agarwala 1977; Sklansky 1978). This equivalence suggests that the HT can simply be seen as an efficient implementation of a convolution operation with optimum results in terms of detection error. Accordingly, by recalling the properties of convolution in the frequency domain, the HT can be interpreted as a filtering process. Extensions of the HT, as for example the generalized Hough transform (GHT) (Ballard 1981), have mainly been based on the idea of template matching. The concepts of template matching and convolution have also been used as the base of a formal definition that suggests that the HT can be related to maximum likelihood estimators (Princen et al. 1992). Other work has shown that the HT is actually a special case of a more general transform developed by Radon in 1917 (Deans 1981). This equivalence provided a formalism that benefits from previous research in the Radon transform. Other generalizations and extensions of the HT have mainly been based on geometric interpretation, which might suggest that the HT is defined based on metric properties of shapes such as distances and angles. The inclusion of metric properties in this definition has provided new ways of using the HT. In a general sense, the HT can also be related to methods of parameter estimation developed in robust statistics (Roth \& Levine 1993).

The motivation behind the development of a variety of interpretations of the HT has been twofold. First, the pragmatic interest of developing procedures capable of shape extraction, and secondly, the establishment of a mathematical definition 
which allows a formal study of the technique and its properties. The main difficulty in the second aspect is that the HT seems to have an ambiguous definition that can be formalized from different points of view, rather than being defined by a unique concept. Thus, the HT has been studied as a particular case of other established techniques. Here, we show that in fact the HT implicitly defines a general concept that can be seen as a convergent point of many techniques used in pattern matching. This paper is not aimed to present an algorithm or an application of the HT, but it is aimed to show how the development of the HT has a dual in ideas previously developed in projective geometry. We show that the definition of the HT in the Euclidean space corresponds to the principle of duality in projective geometry. The correspondence between the definition of the HT and the principle of duality suggests a more general definition of the Hough transform with a deeper meaning in pure geometry. This definition introduces the formalism of projective geometry to shape extraction and analysis and thus the ideas, properties and geometric relationships in the projective space can influence the development of pattern matching techniques.

The principle of duality is one of the most important and elegant concepts in geometry. It was first stated by Gergonne in 1826 and developed in projective geometry by Poncelet, Brianchon, Plücker, Grassmann and others during the 18th and 19th centuries (Veblen \& Young 1910; Plücker 1865; Tuller 1967). Although the most basic form of this principle, which established certain reciprocity or analogy between the definition of a point and a line, makes evident the relationship with the HT, the influence of the principle of duality in the HT and related techniques has not been recognized. In general, geometric concepts have influenced the development of many areas in computer vision. For some concepts such as the use of invariance to recognize three-dimensional models and to derive the geometry of a scene (Mundy \& Zisserman 1992), the relationship between geometry and computer vision has been clearly established and some important results developed in computer vision could be traced as the reformulation of established theorems and properties of geometry (Buchanan 1988). However, in other fields of computer vision the relationship with geometry has not been formally established. In particular, in this paper we focus on the relationship between the principle of duality and the definition of the HT. This relationship not only provides a clearer understanding of the logical foundations of the HT, but also introduces the theorems and analytic methodology of projective geometry to the study of the dual nature of the process of pattern matching. This association extends the generality of the interpretation of the HT.

This paper is divided into six sections. The next section contains a brief introduction to the principle of duality and shows the correspondence between the original definition of the HT and the dual interpretation of a line and a point. Section 3 considers the relationship between the definition of a figure in projective geometry and a shape in pattern matching. This relationship is used to show the correspondence of dual figures in projective geometry and the definition of the HT for shapes composed of simple primitives. Section 4 shows the correspondence between the development of duality for high-dimensional spaces defined by Grassmann coordinates and the extensions of the HT to conics. Section 5 considers the definition of duality for parametric forms. Parametric forms are used to develop a dual analytic expression of general forms under affine and projective transformations. Finally, $\S 6$ presents conclusions. 


\section{Duality}

Geometry studies the properties of interrelated elements or figures in a space. Here, we are interested in the meaning of descriptive relationships and properties when elements are related to points and curves in images. We are particularly interested in the descriptive properties of collinearity and concurrence (Tuller 1967). These properties are defined by a relationship called incidence, which in its simplest form is applied to a point and a line. When a point and a line are incident, we say that the point lies on the line, or the line contains the point. Likewise, a point and a plane are called incident when a point lies on the plane or the plane contains the point. Collinearity is defined when points are incident with the same line, while concurrence is defined when lines are incident with the same points. These relationships can be also established between points in higher dimensions and hyperplanes. The relationship between the principle of duality for hyperplanes, and the generalized form of the HT, will be discussed in $\S \S 4$ and 5 .

The relationship of incidence defines a series of theorems (the propositions of incidence) that reveal a certain duality in a plane. This duality requires the assumption that any two lines in a plane can be defined as concurrent. Thereby, two points always determine a line (i.e. collinear points) and two lines always determine a point (i.e. concurrent lines). As discussed in Baker (1971) the propositions of incidence are not consistent with Euclidean geometry. In the Euclidean space, duality is not completely valid due to the fact that the postulate of parallel lines allows us to define two non-concurrent lines. Perhaps this is the reason why duality was not recognized until the development of projective geometry during the 18th and 19th centuries. In this geometry, the concept of parallel lines is removed, so all lines intersect. Parallel lines in the Euclidean plane intersect at infinity in the projective plane. This intersection is named an 'ideal' point and all 'ideal' points form an 'ideal' line. Since all ideal points are included in the projective space, then duality is completely defined in projective geometry. Although duality is not completely defined in the Euclidean space, relationships that do not contain ideal points can be represented in the Euclidean space (Baker 1971). In $\S 2 b$ we shall show that the reinterpretation of incidence, according to the principle of duality in the Euclidean space, defines the HT.

\section{(a) The principle of duality}

The principle of duality constitutes an important concept for understanding geometric relationships in the projective space. In geometry, the main significance of this principle is that theorems of points and lines can be proved simultaneously. That is, once a theorem has been proved for points, then a dual theorem for lines can be expressed automatically and vice versa. Here, our main concern centres on showing that the interpretation of the principle of duality has an important role in pattern matching. In order to develop this concept in algebraic terms it is necessary to introduce the analytic geometry of the projective plane. This analytic geometry is developed via homogeneous (or redundant) coordinates. We shall consider homogeneous coordinates as the result of a transformation or correspondence rule between the Euclidean and projective spaces.

Homogeneous coordinates (Pedoe 1963) provide a mechanism for defining geometric relationships under projection. In general, these coordinates can be formed by $n$ independent points defining an $n$-dimensional space. Homogeneous coordinates in 
the three-dimensional projective space will be referred to as Plücker coordinates and they can be mapped into two-dimensional Euclidean space. Coordinates on spaces of higher dimensions will be denoted as Grassmann coordinates. In this section, we limit our study to homogeneous coordinates defining the points of a three-dimensional projective space. These coordinates define the duality between a point and a line.

The central problem of using Euclidean coordinates as an analytical base for projective geometry is that it is not possible to assign coordinates to ideal points. The projective space must include the intersection of two parallel lines as a point, a point which by intuition should be at infinity. Consequently, in order to represent an analytic construction of the projective space, the Euclidean space must be augmented to include points at infinity. One way of augmenting the two-dimensional Euclidean space to avoid the inconsistency in the intersection of two parallel lines is to introduce an extra coordinate. Thus, coordinates in the projective space correspond to triplets of real numbers. In this case, each triplet defines the homogeneous coordinates of the point. The mapping or correspondence of a point defined in Euclidean coordinates $(x, y)$ into homogeneous coordinates is defined as $\boldsymbol{x}=\left(x_{1}, x_{2}, x_{3}\right)$ for $x_{1}=x, y_{1}=y$ and $x_{3}=1$. According to this mapping the equation of a line in the Euclidean space changes from the form $U_{1} x+U_{2} y+U_{3}=0$ to the homogeneous form

$$
U_{1} x_{1}+U_{2} x_{2}+U_{3} x_{3}=0 .
$$

In this definition, since any coordinate proportional to $\left(x_{1}, x_{2}, x_{3}\right)$ satisfies the equation of a line, then any triplet of the form $\left(a x_{1}, a x_{2}, a x_{3}\right)$, where $a$ is a constant, can also represent the coordinates of the point $(x, y)$. Thus, two parallel lines in the Euclidean space can be defined as $U_{1} x_{1}+U_{2} x_{2}+U_{3} x_{3}=0$ and $U_{1} x_{1}+U_{2} x_{2}+U_{3}^{\prime} x_{3}=0$ for $U_{3} \neq U_{3}^{\prime}$. The intersection of these lines (i.e. $\left.\boldsymbol{U}_{1} \vee \boldsymbol{U}_{2}\right)$ must satisfy $\left(U_{3}-U_{3}^{\prime}\right) x_{3}=0$, which implies that points at infinity are of the form $\left(x_{1}, x_{2}, 0\right)$. The triplet $(0,0,0)$ is proportional to any triplet $\left(x_{1}, x_{2}, x_{3}\right)$ and at the same time it represents a point at infinity. In order to avoid this ambiguity this point is discarded from the coordinate system. Thus, the projective space consists of all the triplets $\left(x_{1}, x_{2}, x_{3}\right)$ for $x_{3} \neq 0$ and the points at infinity $\left(x_{1}, x_{2}, 0\right)$. This space provides a model wherein points are defined by rays that emanate from the origin. Each point on the ray has coordinates proportional to those of a single Euclidean point. Ideal points are contained in the plane defined by the coordinates $x_{1}$ and $x_{2}$ (i.e. $x_{3}=0$ ), and the plane for which $x_{3}=1$ corresponds to the two-dimensional Euclidean space. A point in the Euclidean space can be mapped into any of the points that form a ray in the projective space by $x_{1}=a x, x_{2}=a y, x_{3}=a$, and the points in the space can be mapped back to the Euclidean space by $x=x_{1} / x_{3}$ and $y=x_{2} / x_{3}$.

The introduction of homogeneous coordinates to represent the fundamental elements of geometry reveals a symmetry in the equation of a projective line. This symmetry and the descriptive properties given by the relationship of incidence naturally lead to the principle of duality. The form of the projective line in equation (2.1) is symmetric in the sense that any triplet (i.e. $\left.\left(x_{1}, x_{2}, x_{3}\right)\right)$ defines a point and, at the same time, a line (i.e. $\left.\left[U_{1}, U_{2}, U_{3}\right]\right)$. That is, equation $(2.1)$ can be seen as the equation of a point for fixed $\left(x_{1}, x_{2}, x_{3}\right)$ or as the equation of a line for fixed $\left[U_{1}, U_{2}, U_{3}\right]$. Thus, the terms 'line' and 'point' are indistinguishable, and theorems and relationships between these elements are said to be dual since they have a double significance. Thereby, by considering the incidence of a point in a line in equation (2.1) we can establish the following principle (Baker 1971). 
Principle 2.1. If we have a plane figure consisting of lines, there exists also a figure consisting of points, such that to any point of the first figure laying on a line of that figure, there corresponds a line of the second figure passing through a point of this figure, which corresponds to the line of the first figure.

Principle 2.1 defines a mapping between the fundamental elements of dual figures. Since these elements are defined in terms of incidence, then this principle can be interpreted using the properties of collinearity and concurrence. In the next section we discuss the algebraic form of this descriptive part of the principle of duality and its intimate relationship with the HT.

\section{(b) The dual interpretation of a line and the HT}

The HT has long been recognized as an original concept that can provide an efficient approach to shape extraction. Nevertheless, as we shall show, its formulation corresponds to geometric concepts that can be defined according to the principle of duality. Intuitively, we can observe a palpable analogy between the formulation of the HT and the duality of figures defined in projective geometry. Both the HT and the principle of duality define a dual figure by interpreting points as lines and, conversely, lines as points. In this analogy, descriptive properties of collinearity and concurrence can define relationships of incidence in the dual interpretation. That is, a set of collinear points in an image form a dual interpretation as a set of concurrent lines, while the intersection point of concurrent lines has a dual interpretation as the line that forms the collinear points in the image. That is, in a dual interpretation a line is defined as a point of a set of concurrent lines. In the HT, concurrent lines form a peak (i.e. a point) that defines a point whose dual interpretation corresponds to a line in the image space.

In this analogy between the HT and the principle of duality, the only difference is the generality of the definitions. While the principle of duality is a general definition in the projective space, the HT has been developed for shape extraction in the Euclidean space. In the HT, a figure is defined in the image space and a dual figure is defined in what has been called the Hough space. The use of these two spaces in the HT results from its algorithmic nature, since dual figures must simultaneously be maintained for processing. Nevertheless, the image space and the Hough space have the same mathematical properties delineated by the Euclidean space. This means that in the HT, shapes and their dual interpretations are defined in the Euclidean space which seems to be an important difference from the principle of duality that is expressed in the projective space. However, we must recall that the points in the Euclidean space can be mapped into the projective space and vice versa. Thus, it is possible to derive an interpretation of the principle of duality in the Euclidean space as long as we ensure that ideal points are not mapped from the projective space into the Euclidean space (i.e. without contradicting the axiom that establishes that two lines always meet at a point) (Baker 1971). Here, we shall show that the HT is given by this interpretation of the principle of duality.

In order to study the relationship between the HT and the principle of duality in a formal way, it is necessary to consider an algebraic statement of the dual properties of figures in the projective space and to then translate the analytic equations by mapping the points into the Euclidean space. By following this approach, let us suppose that we have a line that we want to identify only as a point. This is defined by 

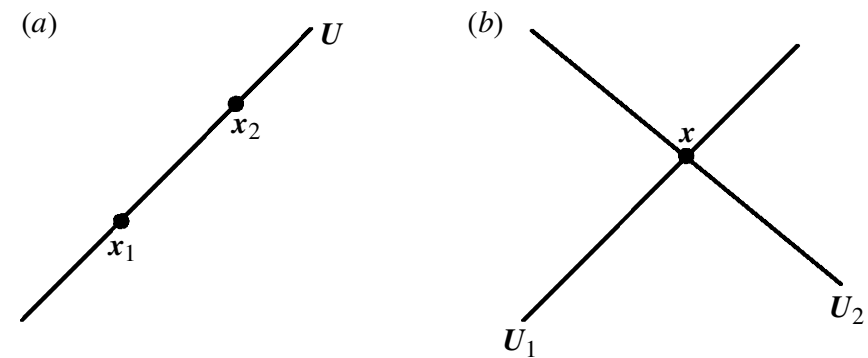

Figure 1. Example of the descriptive relationship defined by the principle of duality: (a) collinear points; (b) concurrent lines.

the dual relationship presented in equation (2.1). Nevertheless, this correspondence is descriptive, which means that the line does not define the position of the point and neither does the point define the position of the line, but the correspondence only determines the existence of incidences. For example, we can establish the dual of the line $\boldsymbol{U}$ in figure $1 a$ as the point $\boldsymbol{x}$ in figure $1 b$; however, the position of the point $\boldsymbol{x}$ cannot be determined by the existence of the line $\boldsymbol{U}$, but it is necessary to consider the descriptive properties given by the concurrence of two lines $\boldsymbol{U}_{1}$ and $\boldsymbol{U}_{2}$ (i.e. by the relationship $\boldsymbol{x}=\boldsymbol{U}_{1} \vee \boldsymbol{U}_{2}$ ). The duals of the lines $\boldsymbol{U}_{1}$ and $\boldsymbol{U}_{2}$ are the collinear points $\boldsymbol{x}_{1}$ and $\boldsymbol{x}_{2}$, respectively. The collinearity property of the points $\boldsymbol{x}_{1}$ and $\boldsymbol{x}_{2}$ is due to the fact that the lines $\boldsymbol{U}_{1}$ and $\boldsymbol{U}_{2}$ define a point $\boldsymbol{x}$ that is dual to the line $\boldsymbol{U}$. Then, the dual of the lines $\boldsymbol{U}_{1}$ and $\boldsymbol{U}_{2}$ must define two collinear points $\boldsymbol{x}_{1}$ and $\boldsymbol{x}_{2}$. That is, $\boldsymbol{x}=\boldsymbol{U}_{1} \vee \boldsymbol{U}_{2}=\left(\boldsymbol{U}_{1}^{*} \vee \boldsymbol{U}_{2}^{*}\right)^{*}=\left(\boldsymbol{x}_{1} \wedge \boldsymbol{x}_{2}\right)^{*}=\boldsymbol{U}^{*}$, where $\vee$ denotes the meet, $\wedge$ denotes the join and the $*$ denotes the dual. This duality between the incidence relationship is a well-known result in projective geometry which, loosely, establishes that concurrence in a figure corresponds to collinearity in its dual. In our notation we have distinguished between points and lines by using lowercase and uppercase; however, mathematically, points and lines are dual and, as such, indistinguishable since points and lines are equally defined by a triad of coordinates. Thus, for convenience, the notation only specifies whether a point or a line is defined by the property of collinearity or concurrence.

The point $\boldsymbol{x}$ in figure $1 b$ is defined as the point of concurrence of the two lines $\boldsymbol{U}_{1}$ and $\boldsymbol{U}_{2}$. If the dual representation of the points $\boldsymbol{x}_{1}=\left(x_{1,1}, x_{1,2}, x_{1,3}\right)$ and $\boldsymbol{x}_{2}=\left(x_{2,1}, x_{2,2}, x_{2,3}\right)$ are the lines $\boldsymbol{U}_{1}=\left[U_{1,1}, U_{1,2}, U_{1,3}\right]$ and $\boldsymbol{U}_{2}=\left[U_{2,1}, U_{2,2}, U_{2,3}\right]$, respectively, then the relationship $\boldsymbol{x}=\boldsymbol{U}_{1} \vee \boldsymbol{U}_{2}$ is given by the solution of two linear equations. For a point $\boldsymbol{x}=\left(x_{1}, x_{2}, x_{3}\right)$ and a line $\boldsymbol{U}=\left[U_{1}, U_{2}, U_{3}\right]$, we have

$$
U_{1,1} x_{1}+U_{1,2} x_{2}+U_{1,3} x_{3}=0, \quad U_{2,1} x_{1}+U_{2,2} x_{2}+U_{2,3} x_{3}=0 .
$$

That is,

$$
\boldsymbol{x}=\left(\left|\begin{array}{ll}
U_{1,2} & U_{1,3} \\
U_{2,2} & U_{2,3}
\end{array}\right|,\left|\begin{array}{ll}
U_{1,3} & U_{1,1} \\
U_{2,3} & U_{2,1}
\end{array}\right|,\left|\begin{array}{ll}
U_{1,1} & U_{1,2} \\
U_{2,1} & U_{2,2}
\end{array}\right|\right) .
$$

We can obtain a dual representation of this point as a line $\boldsymbol{U}=\left(\boldsymbol{x}_{1} \wedge \boldsymbol{x}_{2}\right)$ that is collinear to the points $\boldsymbol{x}_{1}=\left(x_{1,1}, x_{1,2}, x_{1,3}\right)$ and $\boldsymbol{x}_{2}=\left(x_{2,1}, x_{2,2}, x_{2,3}\right)$ by changing lines by points and points by lines in equation (2.2). That is,

$$
U_{1} x_{1,1}+U_{2} x_{1,2}+U_{3} x_{1,3}=0, \quad U_{1} x_{2,1}+U_{2} x_{2,2}+U_{3} x_{2,3}=0,
$$


whose solution can be expressed as

$$
\boldsymbol{U}=\left[\left|\begin{array}{ll}
x_{1,2} & x_{1,3} \\
x_{2,2} & x_{2,3}
\end{array}\right|,\left|\begin{array}{ll}
x_{1,3} & x_{1,1} \\
x_{2,3} & x_{2,1}
\end{array}\right|,\left|\begin{array}{ll}
x_{1,1} & x_{1,2} \\
x_{2,1} & x_{2,2}
\end{array}\right|\right] .
$$

Equations (2.3) and (2.5) use the relations of incidence to obtain the coordinates of dual elements of equation (2.1). In pattern matching the HT and related techniques have exploited the dual relationship in equation (2.4) to extract shapes from images. In the HT, if $\boldsymbol{U}$ represents a line in an image, then it can be defined as the point of concurrence of a set of lines obtained as the dual of collinear points. That is, for a set of image points $\left\{\boldsymbol{x}_{j}\right\}$, the HT defines the element $\boldsymbol{U}$ whose points of concurrence correspond to collinear elements in $\left\{\boldsymbol{x}_{j}\right\}$. Hence, the HT locates a set of lines in images by using the relations of incidence to obtain the coordinates of dual elements. If $\boldsymbol{x}_{i}=\left(x_{j, 1}, x_{j, 2}, x_{j, 3}\right)$, then the HT solves for the system of equations of the form

$$
U_{1} x_{j, 1}+U_{2} x_{j, 2}+U_{3} x_{j, 3}=0 .
$$

This system formed by all the points $\left\{\boldsymbol{x}_{j}\right\}$ is over-determined. To solve the system we need only two equations. However, in images the set $\left\{\boldsymbol{x}_{j}\right\}$ contains points due to noise, as well as those from the target shape, so the problem focuses on identifying the best solution for the system of equations. In the HT, the solution is computed by counting the number of times that a line $\boldsymbol{U}$ is defined by an equation in the system. That is, the HT searches for the solution that satisfies a larger number of equations simultaneously. This strategy is robust and has been demonstrated to have good performance when data is contaminated by noise and/or is occluded. If we consider each line of the form in equation (2.6) for fixed $\boldsymbol{x}_{j}$, then the solution of the system is given by the point $\boldsymbol{U}_{i}$, which is a valid solution for the majority of the equations. That is, the HT result is the maximum of the expression

$$
\mathrm{HT}\left(\boldsymbol{U}_{i}\right)=\sum_{j} D\left(\boldsymbol{U}_{i}, \boldsymbol{x}_{j}\right)
$$

for a function

$$
D\left(\boldsymbol{U}_{i}, \boldsymbol{x}_{j}\right)= \begin{cases}1, & \text { if } U_{i, 1} x_{j, 1}+U_{i, 2} x_{j, 2}+U_{i, 3} x_{j, 3}=0 \\ 0, & \text { elsewhere }\end{cases}
$$

Equation (2.7) provides a computational way of obtaining the solution of equation (2.6) and there exists a direct equivalence between equations (2.4) and (2.6). Accordingly, the HT definition provides a computational approach to obtain the dual elements defined in projective geometry and what is named the Hough space is actually the dual form of the projective space.

In equation (2.7) we solve for the values of $\boldsymbol{U}$ given the values of $\boldsymbol{x}$, thus the values of $\boldsymbol{U}$ can be interpreted as a set of parameters of the line. These parameters are defined in the projective space and can be reinterpreted in the Euclidean space by recalling the relationship between the Euclidean space and the projective space discussed in $\S 2 a$. Thus, we can obtain a reparametrization by projecting the coordinates of $\boldsymbol{U}$ in the Euclidean space (Eckhardt \& Manderlechner 1990). That is, by using a normalization constraint to eliminate the redundancy in homogeneous coordinates. The elimination of homogeneous coordinates changes the general form 
of duality in the projective space to a particular form in the Euclidean space. In general, a parametrization can be defined by

$$
A_{1} x_{1}+A_{2} x_{2}+A_{3} x_{3}=0
$$

for a transformation of the form

$$
A_{1}=T_{1}\left(U_{1}, U_{2}, U_{3}\right), \quad A_{2}=T_{2}\left(U_{1}, U_{2}, U_{3}\right), \quad A_{3}=T_{3}\left(U_{1}, U_{2}, U_{3}\right) .
$$

For example, for $A_{1}=U_{1} / U_{2}, A_{2}=1$ and $A_{3}=U_{3} / U_{2}$ and by mapping the point $\left(x_{1}, x_{2}, x_{3}\right)$ into the Euclidean space using the relationships $x=x_{1} / x_{3}$ and $y=x_{2} / x_{3}$ defined in $\S 2 a$, we observe that the definition in equation (2.7) is the same definition of the Hough transform originally presented in Hough (1962) and Rosenfeld (1969). That is, equation (2.6) becomes

$$
A_{1} x+y+A_{3}=0 .
$$

In this definition, we suppose that points exist at infinity in Euclidean space and these points are obtained when $U_{2}$ is zero. For $m=-A_{1}$ and $b=-A_{3}$ this equation can be written as the general form of the line that is used in the original definition of the HT (Hough 1962; Rosenfeld 1969). That is, $y-m x-b=0$. Thus equation (2.7) can be rephrased as

$$
\mathrm{HT}(m, b)=\sum_{j} D\left(m, b, x_{j}, y_{j}\right),
$$

which corresponds to the definition of the HT (Princen et al. 1992). Accordingly, the dual point $\left(U_{1}, U_{2}, U_{3}\right)$ can be interpreted in the Euclidean space as a point defined by the parameters $m$ and $b$. The line $\boldsymbol{A}$ relates each dimension of the projective space to the parameters of the general form of the line. Thus, the principle of duality can be interpreted as a mapping between image points and the parameters of a line in such a way that equation (2.6) defines a set of loci in the parameter space whose intersection or concurrent point defines the parameters of a line.

Other projections can be used to obtain alternative parametrizations of shapes. These parametrizations have different properties. For example, the previous parametrization allows the identification of lines in an image with the same slope as collinear points in the dual space (Wahl 1989). The normal parametrization provides a bounded dual space (Duda \& Hart 1973) and it provides a symmetric correspondence of a figure with itself, thus features in images and dual spaces can easily be related. The normal parametrization is given by

$$
A_{1}=-\frac{U_{1}}{U_{1}^{2}+U_{2}^{2}}, \quad A_{2}=-\frac{U_{2}}{U_{1}^{2}+U_{2}^{2}}, \quad A_{3}=1 .
$$

By mapping the point $\left(x_{1}, x_{2}, x_{3}\right)$ into the Euclidean space, equation (2.9) becomes

$$
A_{1} x+A_{2} y+1=0,
$$

which corresponds to the normal parametrization $c x+d y+1=0$. In this case, $c$ and $d$ define the components of a vector perpendicular to the line in equation (2.11) and passing through the origin. In conclusion the mapping of the HT is defined by the correspondence given by the principle of duality. The definition of the HT for different parametric forms can be obtained by considering the principle of duality in Euclidean space obtained by different parametrizations. 


\section{Figures and shapes}

\section{(a) Duality of figures and shapes}

In the previous discussion, we considered the analytic form of the relationships of incidence in the principle of duality for plane-analytic projective geometry. This allowed us to establish a relationship between the HT and the dual definition of a line and a point. However, a more general approach, the analysis of images, requires the study of elaborate shapes capable of representing complex world structures. In pattern matching there are two possible ways of representing complex shapes. First, shapes can be defined as being composed of simple elements and secondly they can be characterized by a more complex equation. In this section we consider the definition of shapes composed of several elements and its relationship to concepts developed in geometry. The extension of the HT to complex shapes is considered in $\S \S 4$ and 5 .

We can observe an evident relationship between the concept of a shape composed of elements and the concept of a figure developed in projective geometry. However, the properties and studies in both disciplines have as yet been unrelated. The aim of this section is to show the relationship between the concepts of shape and figure.

We can relate the concept of shape, used in pattern matching, with the concept of figure used in geometry. Based on this relationship, we can observe that the extensions of the HT to polygonal shapes can be related to the definition of duality of figures in projective geometry. In geometry, a figure is defined as a set of points, lines and planes in space (Veblen \& Young 1910). Since each of these elements has a dual representation, then a whole figure ought to have a dual definition. Paraphrasing, in a general form the principle of duality establishes an equivalence of figures defined as a collection of the fundamental geometric elements. That is, for a figure $F=\left\{\boldsymbol{U}_{i}\right\}$ we can define the dual of each element $\boldsymbol{U}_{i}$ by equation (2.1) to obtain another figure $f=\left\{\boldsymbol{x}_{i}\right\}$. Thus, for any figure there exists a corresponding dual figure defined by the dual of each element that defines the first figure. If we consider this general form of duality, then a dual representation of a shape can be obtained by considering that it is composed of a collection of parts. That is, if we relate each part with a geometric element, then the dual of a shape can be defined as the dual of a figure. If we define each straight line forming the sides of a polygon as an element of a shape, then a dual representation can be obtained by considering the dual form of each line. In this way, each side can be mapped into the dual space in a point such that a collection of lines and points define the correspondence between a polygon and its dual figure.

\section{(b) One-to-one reciprocity}

If a figure is equivalent to a shape, then properties of clusters of elements of figures and shapes must be the same. However, the property of uniqueness seems to be contradictory between the development of the HT and the principle of duality. While a well-known result in projective geometry establishes that the dual of a figure is unique, a well-known result in pattern matching (Rosenfeld \& Weiss 1995) shows that the HT of a shape can be non-unique. We discuss the extent to which the oneto-one reciprocity of the principle of duality is congruent with the results derived in the study of the HT.

The relationship in equation (2.1) defines a one-to-one reciprocal correspondence between two elements (Veblen \& Young 1910). Given one element $\boldsymbol{x}$, this relationship 
(a)

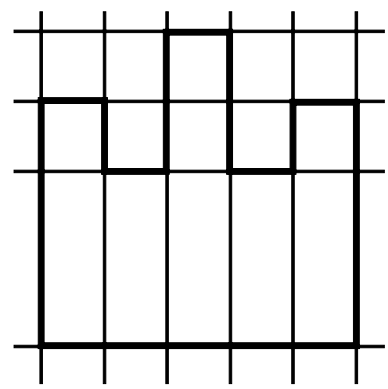

(b)

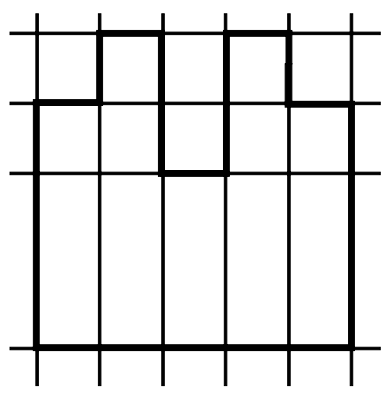

Figure 2. Example of two shapes with the same dual representation.

defines a unique dual element $\boldsymbol{U}$ in such a way that $\boldsymbol{U}$ has a unique corresponding element $\boldsymbol{x}$. In consequence, the correspondence between the collection of elements $F=\left\{\boldsymbol{U}_{i}\right\}$ and $f=\left\{\boldsymbol{x}_{i}\right\}$ is one-to-one. That is, every element of a figure $F$ corresponds to a unique element of $f$ in such a way that every element in $f$ is the correspondent of a unique element of $F$. This well-known property of dual figures and the fact that the HT defines the correspondence between figures for lines and points according to the principle of duality, can lead to the assertion that the mapping obtained by the HT is actually unique. However, research in pattern matching has shown that two concave polygons can produce the same cluster pattern in the dual space (Rosenfeld \& Weis 1995). This discrepancy would appear to be contradictory to the equivalence between the principle of duality and the HT, but it can be explained by the difference between the interpretation of a figure and a shape.

The bold lines in figure 2 show an example of two polygons that have the same arrangement of points in the dual space. In this example a shape is defined by a collection of lines that delineate the sides of the polygon (non-bold lines in figure 2). We can observe that since both shapes define the same lines, then it is evident that the dual figure obtained by applying the dual relationship in equation (2.6) to each line is the same. The dual elements of the lines in figure $2 a, b$ define the points in figure $3 a, b$, respectively. The points in figure $3 c, d$ define the dual figure according to the mapping in equation (2.13). It is important to observe that in this example the dual definition is unique, but the definition of a figure from a shape is not. Since two different shapes define the same figure, then the mapping produces an ambiguous representation of the polygon. Accordingly, we can argue that the HT, as the principle of duality, defines a one-to-one mapping between two figures and that the definition of a polygon as a collection of the lines defined by its sides does not define a unique figure. That is, the definition of a polygon as a collection of lines is ambiguous and not the mapping defined by the HT. In more formal terms, we can say that if we define two figures $F_{1}$ and $F_{2}$ such that the corresponding dual elements are equal (i.e. $f_{1}=f_{2}$ ), then by the uniqueness property of duality we have $F_{1}=F_{2}$. Thus, if for two different shapes $S_{1}$ and $S_{2}$ we define the two figures $F_{1}=R\left(S_{1}\right)$ and $F_{2}=R\left(S_{2}\right)$, whose dual figures are $f_{1}=D\left(F_{1}\right)$ and $f_{2}=D\left(F_{2}\right)$, respectively, then if $f_{1}=f_{2}$ we have $R\left(S_{1}\right)=R\left(S_{2}\right)$, while the mapping $D$ is unique. This is the case in the example of figure 2 since the elements defined by the non-bold lines imply that the figures are the same. 


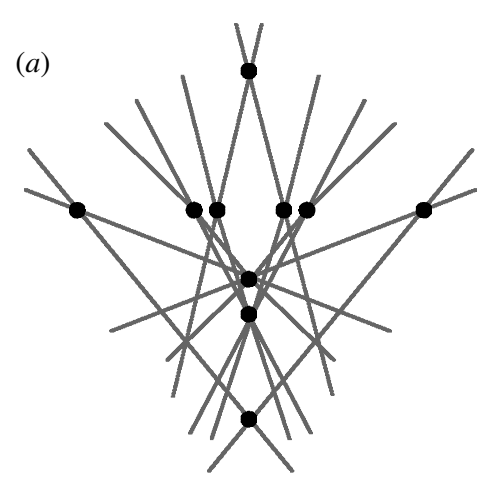

(c)

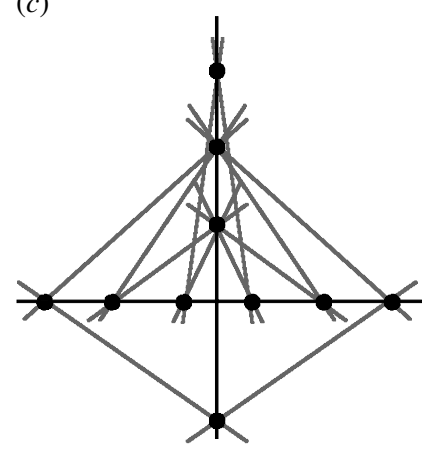

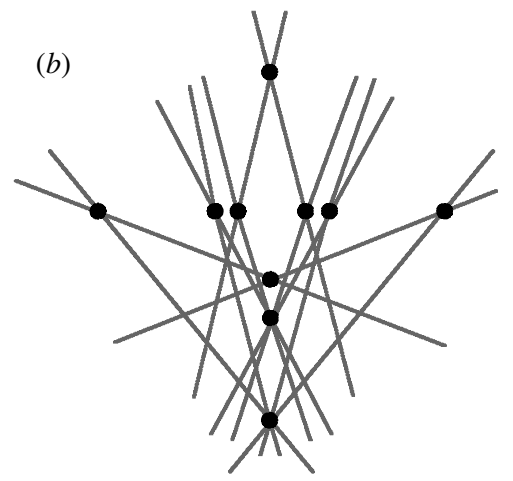

(d)

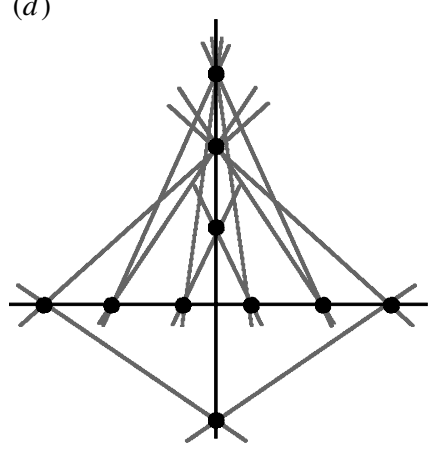

Figure 3. Dual representation of the shapes in figure 2: (a) slope-intercept parametrization for figure $2 a ;(b)$ slope-intercept parametrization for figure $2 b ;(c)$ normal parametrization for figure $2 a ;(d)$ Normal parametrization for figure $2 b$.

In order to identify a polygon with a unique figure it is necessary to consider a mapping that defines different figures for different polygons. This can be achieved by considering a polygon as a figure composed of lines and points. Since a figure consisting of lines and points defines a dual figure consisting of points and lines (Baker 1971), then the dual of a polygon is a shape obtained by mapping vertices as sides, and sides as vertices. In this case, sides and vertices define a unique figure and the HT defines a one-to-one representation. This dual representation has been used in computer vision to study the projection of polyhedral shapes in images (Wahl 1989; Stahs \& Wahl 1989). Figure 3 shows a dual interpretation for the polygons in figure 2. The lines in the figure were obtained by mapping points using equation (2.5). Here, we can observe that although the mappings of lines define the same points, the mappings of points define different lines and the duals are different. Accordingly, the HT and the principle of duality define a one-to-one mapping and the representation of a polygon as a set of lines is ambiguous. Thus, other geometric elements also have an interpretation in the dual space. We can also notice that the form of a figure in a dual space is unique in projective space, but it changes its appearance when it is mapped into the Euclidean space under different parametrizations. However, it can be uniquely identified by the properties of collinearity and concurrence. By way of illustration, figure 4 shows an example of the mapping of dual elements in an image. The image in figure $4 a$ contains the polygons defined in figure 2. Figure $4 b$ shows a 
(a)

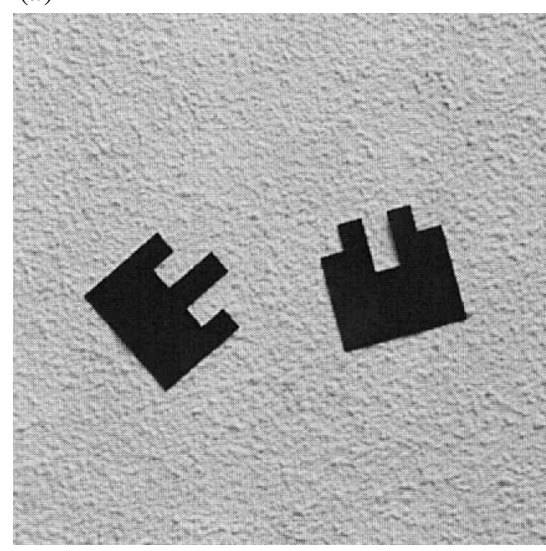

(b)

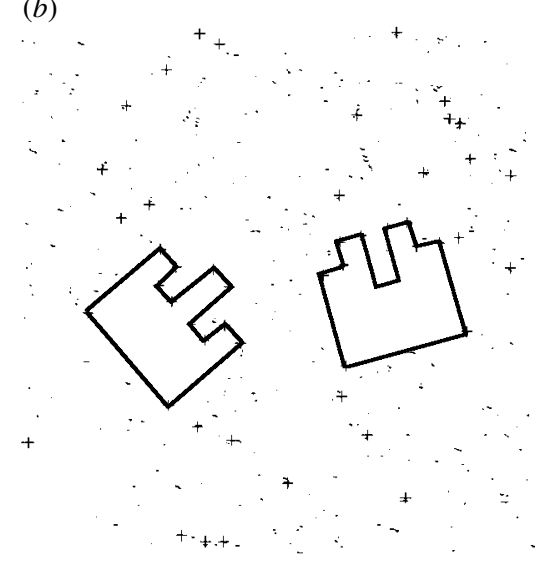

(c)

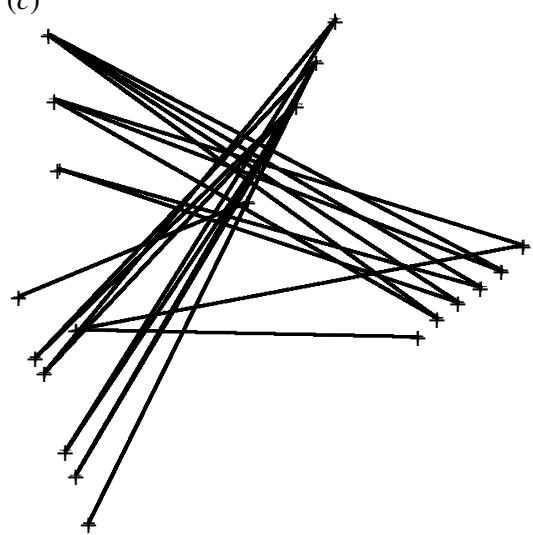

Figure 4. Example of the mapping of dual elements: (a) image;

(b) located edges and points; (c) dual space.

collection of edges and corner points obtained by applying local operators (Canny 1983; Harris \& Stephens 1988). In this figure, points are indicated by a ' +' symbol to distinguish them from edges. Each element defines a relationship of the form in equation (2.1) for points and lines. Thus, to obtain the dual representation it is necessary to solve for a pair of systems of equations similar to equations (2.2) and (2.4). Figure $4 c$ shows the dual representation of the elements in figure $4 b$. Each point in figure $4 c$ solves for a large number of equations simultaneously and represents a side of the polygon. We obtained the dual form by eliminating incompatible equations in neighbourhoods that were progressively increased in size. Only compatible solutions were considered in further steps. The points in figure $4 c$ were obtained by parametrizing the solution in the normal form given in equation (2.13). The lines in figure $4 c$ represent the corners of the polygons in figure $4 b$. It can be seen that collinear points correspond to parallel lines in the Euclidean space, which are concurrent at infinity in the projective space. The lines in figure $4 c$ were obtained by considering for each line given by each combination of points in figure $4 c$ the corresponding point in figure $4 b$. If the local operator in the figure had located the point, then a line in the dual space 
was defined. In general, points define continuous or discrete curves, and we are interested in identifying a particular arrangement in the primal and dual space. The next two sections consider how points forming curves are mapped in the dual space. This shows the equivalence between the principle of duality and extensions to the HT.

\section{Duality in multidimensional projective spaces}

(a) Multidimensional coordinates

Research in the HT has extended its definition to the extraction of shapes represented by quadratic forms such as circles and ellipses (Duda \& Hart 1972). In this case the dual form defines a single point in the dual space. In this section we shall show that these extensions correspond to the principle of duality of geometry for points and lines represented by coordinates with an increased number of dimensions (Grassmann coordinates).

Equation (2.1) establishes the duality between a point and a line for plane analytic projective geometry. In this equation, the parallel postulate is removed by representing points and lines as triplets. To generalize this, it is natural to develop homogeneous coordinates that remove the parallel postulate for planes or hyperplanes in the Euclidean space. These coordinates are referred to as Grassmann coordinates and represent points and hyperplanes in projective spaces with an arbitrary number of dimensions. For example, four-tuples can represent a point in a four-dimensional projective space. The first three coordinates are considered to be proportional to the Euclidean space coordinates, while the fourth coordinate defines the proportionality constant. That is, an ordinary point with Euclidean coordinates $p_{\mathrm{e}}=(x, y, z)$ can be defined in homogeneous coordinates as $p_{\mathrm{h}}=\left(x_{1}, x_{2}, x_{3}, x_{4}\right)$ for $x_{1}=a x, y_{1}=a y$, $x_{3}=a z$ and $x_{4}=a$. In this case, a point at infinity is distinguished by a value of zero in the fourth coordinate.

According to this definition the equation of a plane in the Euclidean space changes from the form $U_{1} x+U_{2} y+U_{3} z+U_{4}=0$ to the homogeneous form

$$
U_{1} x_{1}+U_{2} x_{2}+U_{3} x_{3}+U_{4} x_{4}=0 .
$$

The symmetry of this equation defines a dual relationship between a point $\left(x_{1}, x_{2}\right.$, $\left.x_{3}, x_{4}\right)$ and a plane $\left[U_{1}, U_{2}, U_{3}, U_{4}\right]$, and it states that a point and a plane can be described by the same equation. Hence, the terms point and plane are indistinguishable and we can generalize the principle of duality to define a mapping in space coordinates. Thus, by considering the relationships of incidence, we have that the problems of finding the plane through three non-collinear points and finding the intersection of three non-collinear planes are dual. In analytic terms these problems define three homogeneous linear equations in four variables (Fishback 1969).

For hyperplanes, equations (2.1) and (4.1) can be generalized to the homogeneous equation

$$
\sum_{k=1}^{n} U_{k} x_{k}=0
$$

for the $n$-tuples $\left(x_{1}, x_{2}, \ldots, x_{n}\right)$ and $\left[U_{1}, U_{2}, \ldots, U_{n}\right]$. Since in this generalization the relations of incidence are maintained, a general form of the principle of duality establishes the equivalence between points and planes of arbitrary dimensions. 


\section{(b) The dual interpretation of a hyperplane and the HT}

In the same way that the HT defines the correspondence between lines and points in the projective plane according to the principle of duality, it is possible to show that the extension of the HT for quadratic equations is a particular form of the generalization of the principle of duality to higher dimensions. The equation of a conic, $A x^{2}+B y^{2}+C x y+D x+E y+F=0$, can be expressed in homogeneous form as

$$
U_{1} x_{1}+U_{2} x_{2}+U_{3} x_{3}+U_{4} x_{4}+U_{5} x_{5}+U_{6} x_{6}=0,
$$

by considering that

$$
x_{1}=a x^{2}, \quad x_{2}=a y^{2}, \quad x_{3}=a x y, \quad x_{4}=a x, \quad x_{5}=a y, \quad x_{6}=a .
$$

In an analogous way to the mapping of a point $(x, y)$ on a line into a point in a plane of the projective space by the relationships $x_{1}=a x, x_{2}=a y$ and $x_{3}=a$, the definitions in equation (4.4) map a point $(x, y)$ in a conic into a point on a hyperplane in the projective space. Thus, all the points that form a conic in the Euclidean plane can be seen as a point or a hyperplane in the six-dimensional projective space.

Equation (4.3) defines a dual descriptive relationship between a point $\boldsymbol{x}=\left(x_{1}, x_{2}\right.$, $\left.\ldots, x_{6}\right)$ and the hyperplane $\boldsymbol{U}=\left(U_{1}, U_{2}, \ldots, U_{6}\right)$. In order to define the positions of the point and of the hyperplane we can consider the concurrence of five hyperplanes at one point or the dual defined by the six coplanar points, that is, by solving simultaneously five homogeneous linear equations in six variables. If $\sum_{k=1}^{6} U_{j, k} x_{k}=0$ is the equation of the $j$ th plane, then a point $\boldsymbol{x}$ is given by

$$
\begin{aligned}
\left(x_{1}, x_{2}, \ldots, x_{6}\right)= & \left(\left|\begin{array}{cccc}
U_{1,2} & U_{1,3} & \ldots & U_{1,6} \\
U_{2,2} & U_{2,3} & \ldots & U_{2,6} \\
\vdots & \vdots & \ldots & \vdots \\
U_{6,2} & U_{6,3} & \ldots & U_{6,6}
\end{array}\right|,\right. \\
& \left.-\left|\begin{array}{cccc}
U_{1,1} & U_{1,3} & \ldots & U_{1,6} \\
U_{2,1} & U_{2,3} & \ldots & U_{2,6} \\
\vdots & \vdots & \ldots & \vdots \\
U_{6,1} & U_{6,3} & \ldots & U_{6,6}
\end{array}\right|, \ldots,\left|\begin{array}{cccc}
U_{1,1} & U_{1,2} & \ldots & U_{1,5} \\
U_{2,1} & U_{2,2} & \ldots & U_{2,5} \\
\vdots & \vdots & \ldots & \vdots \\
U_{6,1} & U_{6,2} & \ldots & U_{6,5}
\end{array}\right|\right) .
\end{aligned}
$$

The dual solution is given by changing points by hyperplanes and hyperplanes by points. Thus, if $\sum_{k=1}^{6} U_{k} x_{j, k}=0$ is the equation of the $j$ th point, then the hyperplane is given by

$$
\begin{aligned}
& {\left[U_{1}, U_{2}, \ldots, U_{6}\right]=\left[\begin{array}{cccc}
x_{1,2} & x_{1,3} & \ldots & x_{1,6} \\
x_{2,2} & x_{2,3} & \ldots & x_{2,6} \\
\vdots & \vdots & \ldots & \vdots \\
x_{6,2} & x_{6,3} & \ldots & x_{6,6}
\end{array} \mid\right.} \\
& \left.-\left|\begin{array}{cccc}
x_{1,1} & x_{1,3} & \ldots & x_{1,6} \\
x_{2,1} & x_{2,3} & \ldots & x_{2,6} \\
\vdots & \vdots & \ldots & \vdots \\
x_{6,1} & x_{6,3} & \ldots & x_{6,6}
\end{array}\right|, \ldots,\left|\begin{array}{cccc}
x_{1,1} & x_{1,2} & \ldots & x_{1,5} \\
x_{2,1} & x_{2,2} & \ldots & x_{2,5} \\
\vdots & \vdots & \ldots & \vdots \\
x_{6,1} & x_{6,2} & \ldots & x_{6,5}
\end{array}\right|\right] .
\end{aligned}
$$


The HT exploits the relationship in equation (4.3) to compute the dual representation of a conic as a point in a hyperplane. A set of image points $\left\{\boldsymbol{x}_{j}\right\}$ defines a collection of hyperplanes $\boldsymbol{U}$ whose point of concurrence corresponds to the hyperplanes that solve equation (4.3). Thus, the generalization of the HT to conic sections generalizes equation (2.6) to a set of simultaneous equations of the form

$$
U_{1} x_{j, 1}+U_{2} x_{j, 2}+U_{3} x_{j, 3}+U_{4} x_{j, 4}+U_{5} x_{j, 5}+U_{6} x_{j, 6}=0 .
$$

As equation (2.6), this equation is over constrained and the HT provides a technique wherein the solution is computed by counting the number of times that a point is defined by an equation in the system. To define the HT according to equation (2.7), it suffices to rewrite equation (2.8) in terms of equation (4.6) instead of equation (2.6). That is,

$$
D\left(\boldsymbol{U}_{i}, \boldsymbol{x}_{j}\right)= \begin{cases}1, & \text { if } \sum_{k=1}^{6} U_{i, k} x_{j, k}=0 \\ 0, & \text { elsewhere. }\end{cases}
$$

Thus, the extension of the HT for conic sections simply defines a way of computing the correspondence between dual figures in projective spaces of dimension six (or five in the projection into the Euclidean space). The dual point $\boldsymbol{U}$ corresponds to the parameters that define a conic in the Euclidean space, which are defined by the concurrent relationship of the principle of duality.

\section{General forms}

\section{(a) Duality of parametric forms}

In a further generalization of the relationship between the principle of duality and the HT we can consider forms which are more general than quadratics. In pattern matching, the interest in this generalization has been motivated by the complex appearance of most objects. Since the HT defines a correspondence between figures according to the principle of duality, then we can show that the analytic definition of the generalization of the HT to arbitrary shapes corresponds to the computation of the dual form in equation (4.2).

In the previous sections we used the notation $\left\{\boldsymbol{x}_{j}\right\}$ to represent a collection of points. In an alternative way, we can express a collection of points as a parametric function $\boldsymbol{x}(j)$. Thus, a point in the Euclidean space is defined by a pair of orthogonal functions of the form $\boldsymbol{x}(j)=(x(j), y(j))$. In general the sequence of points $\boldsymbol{x}(j)$ defines a curve and we are interested in the representation of its dual form which corresponds to the generalization of the HT for arbitrary shapes.

Without loss of generality, we can define a curve $\boldsymbol{x}(j)$ as a linear combination of basis functions $x_{k}(j)$ and $y_{k}(j)$. That is,

$$
x(j)=\sum_{k=1}^{n} U_{k}^{x} x_{k}(j), \quad y(j)=\sum_{k=1}^{n} U_{k}^{y} y_{k}(j) .
$$

Then a homogeneous form of $\boldsymbol{x}(j)$ is given by

$$
\sum_{k=1}^{n+1} U_{k}^{x} x_{k}(j)=0, \quad \sum_{k=1}^{n+1} U_{k}^{y} y_{k}(j)=0
$$


for $U_{n+1}^{x}=1, U_{n+1}^{y}=1, x_{n+1}(j)=-x(j)$ and $y_{n+1}(j)=-y(j)$. In a way analogous to equation (4.5), we can observe that the set of functions $x_{k}(j)$ and $y_{k}(j)$ map each point $(x(j), y(j))$ that defines the collection $\boldsymbol{x}(j)$, into the points

$$
\left(x_{1}(j), x_{2}(j), \ldots, x_{n+1}(j)\right) \text { and }\left(y_{1}(j), y_{2}(j), \ldots, y_{n+1}(j)\right)
$$

in a hyperplane in the projective space. According to equation (4.2), the dual of these elements is given by the elements

$$
\left(U_{1}^{x}(j), U_{2}^{x}(j), \ldots, U_{n+1}^{x}(j)\right) \text { and }\left(U_{1}^{y}(j), U_{2}^{y}(j), \ldots, U_{n+1}^{y}(j)\right),
$$

respectively.

Generalizing our previous development, the dual elements can be determined by the concurrence of $n$ hyperplanes defined by $n$ points $(x(j), y(j))$. If $\left(x\left(j_{m}\right), y\left(j_{m}\right)\right)$ represents the $m$ th point

$$
\begin{aligned}
& {\left[U_{1}^{x}, U_{2}^{x}, \ldots, U_{n+1}^{x}\right]=\left[\begin{array}{cccc}
x_{2}\left(j_{1}\right) & x_{3}\left(j_{1}\right) & \ldots & x_{n}\left(j_{1}\right) \\
x_{2}\left(j_{2}\right) & x_{3}\left(j_{2}\right) & \ldots & x_{n}\left(j_{2}\right) \\
\vdots & \vdots & \ldots & \vdots \\
x_{2}\left(j_{n}\right) & x_{3}\left(j_{n}\right) & \ldots & x_{n}\left(j_{n}\right)
\end{array} \mid\right.} \\
& \left.-\left|\begin{array}{cccc}
x_{1}\left(j_{1}\right) & x_{3}\left(j_{1}\right) & \ldots & x_{n}\left(j_{1}\right) \\
x_{1}\left(j_{2}\right) & x_{3}\left(j_{2}\right) & \ldots & x_{n}\left(j_{2}\right) \\
\vdots & \vdots & \ldots & \vdots \\
x_{1}\left(j_{n}\right) & x_{3}\left(j_{n}\right) & \ldots & x_{n}\left(j_{n}\right)
\end{array}\right|, \ldots,\left|\begin{array}{cccc}
x_{1}\left(j_{1}\right) & x_{2}\left(j_{1}\right) & \ldots & x_{n-1}\left(j_{1}\right) \\
x_{1}\left(j_{2}\right) & x_{2}\left(j_{2}\right) & \ldots & x_{n-1}\left(j_{2}\right) \\
\vdots & \vdots & \ldots & \vdots \\
x_{1}\left(j_{n}\right) & x_{2}\left(j_{n}\right) & \ldots & x_{n-1}\left(j_{n}\right)
\end{array}\right|\right]
\end{aligned}
$$

and $\left[U_{1}^{y}, U_{2}^{y}, \ldots, U_{n+1}^{y}\right]$ is defined by a similar expression. For points defined in images, the system of equations obtained is over-constrained and the HT provides a solution by generalizing the definition in equation (2.7) according to equation (5.2). That is,

$$
D\left(\boldsymbol{U}_{i}, \boldsymbol{x}_{j}\right)= \begin{cases}1, & \text { if } \sum_{k=1}^{n} U_{i, k}^{x} x_{k}(j)=0 \text { and } \sum_{k=1}^{n} U_{i, k}^{y} y_{k}(j)=0, \\ 0, & \text { elsewhere }\end{cases}
$$

which corresponds to the dual element of a general curve $\boldsymbol{x}(j)$. That is, a curve is at the same time represented by $\boldsymbol{x}(j)$ and by the dual $\boldsymbol{U}$ that is given by the maximum value $\boldsymbol{U}_{i}$ in equation (2.7) for $D\left(\boldsymbol{U}_{i}, \boldsymbol{x}_{j}\right)$ defined in equation (5.4). The HT provides a computational model for establishing this dual relationship in images. It is important to notice that the dimension of the elements $\boldsymbol{U}_{i}$ is independent of the form of the curve $\boldsymbol{x}(j)$ but it depends on the linear combination in equation (5.2). As we shall discuss in the next section, equation (5.1) can be related to the parametrization of the curve obtained by applying a transformation that, in pattern matching, models the appearance of an object in the space.

As an example of the dual definition of a general form we can consider the curve in figure $5 a$. The curve is defined for the point given by $\sum_{k=1}^{3} U_{k}^{x} x_{k}(j)=0$ and $\sum_{k=1}^{3} U_{k}^{y} y_{k}(j)=0$ for the functions $x_{1}(j)=1, x_{2}(j)=\cos (j), x_{3}(j)=-x(j)$, $y_{1}(j)=1, y_{2}(j)=\sin (2 j)$ and $y_{3}(j)=-y(j)$ for $j \in[0,2 \pi)$. These functions map the points on the curve into the projective space whose dual is given by $\left[U_{1}^{x}, U_{2}^{x}, U_{3}^{x}\right]$ 
(a)

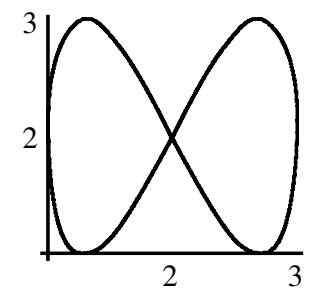

(b)

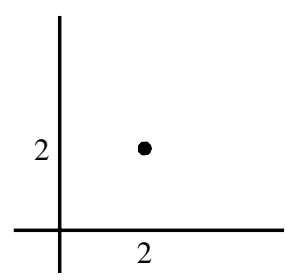

(c)

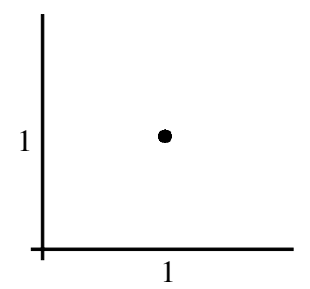

Figure 5. Example of dual space of a general form: $(a)$ curve; $(b)$ projection $\left[U_{1}^{x} / U_{3}^{x}, U_{1}^{y} / U_{3}^{y}\right]$; (c) projection $\left[U_{2}^{x} / U_{3}^{x}, U_{2}^{y} / U_{3}^{y}\right]$.

and $\left[U_{1}^{y}, U_{2}^{y}, U_{3}^{y}\right]$. By the properties of incidence, two equations suffice to determine the dual elements that according to equation (5.3) are given by

$$
\begin{aligned}
& {\left[U_{1}^{x}, U_{2}^{x}, U_{3}^{x}\right]=\left[\left|\begin{array}{ll}
\cos \left(j_{1}\right) & -x\left(j_{1}\right) \\
\cos \left(j_{2}\right) & -x\left(j_{2}\right)
\end{array}\right|,-\left|\begin{array}{ll}
1 & -x\left(j_{1}\right) \\
1 & -x\left(j_{2}\right)
\end{array}\right|,\left|\begin{array}{ll}
1 & \cos \left(j_{1}\right) \\
1 & \cos \left(j_{2}\right)
\end{array}\right|\right],} \\
& {\left[U_{1}^{y}, U_{2}^{y}, U_{3}^{y}\right]=\left[\left|\begin{array}{ll}
\sin \left(2 j_{1}\right) & -y\left(j_{1}\right) \\
\sin \left(2 j_{2}\right) & -y\left(j_{2}\right)
\end{array}\right|,-\left|\begin{array}{ll}
1 & -y\left(j_{1}\right) \\
1 & -y\left(j_{2}\right)
\end{array}\right|,\left|\begin{array}{ll}
1 & \sin \left(2 j_{1}\right) \\
1 & \sin \left(2 j_{2}\right)
\end{array}\right|\right],}
\end{aligned}
$$

for $j_{1}$ and $j_{2}$, any two values that define points on the curve. We can notice that in this parametrization, since $x_{1}(j)=1$ and $y_{1}(j)=1$, the values of $U_{1}^{x}$ and $U_{1}^{y}$ are related to the position of the curve. Figure $5 b, c$ shows the projection of the dual space into two Euclidean planes. The point of each plane shows the projection values $\left[U_{1}^{x} / U_{3}^{x}, U_{1}^{y} / U_{3}^{y}\right]$ and $\left[U_{2}^{x} / U_{3}^{x}, U_{2}^{y} / U_{3}^{y}\right]$, respectively. In a parameter space interpretation used by the HT these values represent the location and size of the curve, respectively.

\section{(b) Forms under geometric transformations}

In the definition of the dual element of a general form in equation (5.2), we can identify a set of $n+1$ functions $x_{k}(j)$ and $y_{k}(j)$ that maps a point in the Euclidean space into the projective space. The definition of these functions is of particular interest in pattern matching, since they provide a meaning to the values of $\boldsymbol{U}_{k}^{x}$ and $\boldsymbol{U}_{k}^{y}$ related to parameters of the transformation that reflects the changes of an object in space, hence controlling its appearance. For example, by considering $x_{1}(j)=1$ and $y_{1}(j)=1$, then the values of $\left[U_{1}^{x} / U_{n+1}^{x}, U_{1}^{y} / U_{n+1}^{y}\right]$ are directly related to the position or translation of a curve. This can be explained by considering that equation (5.1) defines a parametrization of a particular form $\boldsymbol{z}(j)=(u(j), v(j))$. That is, $\boldsymbol{x}(j)=T(z(j))$. If $T$ represents a translation and a change of scale, we have

$$
\boldsymbol{x}(j)=\left[\begin{array}{ccc}
s_{x} & 0 & c_{x} \\
0 & s_{y} & c_{y} \\
0 & 0 & 1
\end{array}\right]\left[\begin{array}{c}
u(j) \\
v(j) \\
1
\end{array}\right]
$$

where the values $\left(c_{x}, c_{y}\right)$ define a translation point and $\left(s_{x}, s_{y}\right)$ a factor of scale in two orthogonal directions. The homogeneous form of $\boldsymbol{x}(j)$ in equation $(5.2)$ can be written as

$$
U_{1}^{x} x_{1}(j)+U_{2}^{x} x_{2}(j)+U_{3}^{x} x_{3}(j)=0 \quad \text { and } \quad U_{1}^{y} y_{1}(j)+U_{2}^{y} y_{2}(j)+U_{3}^{y} y_{3}(j)=0 .
$$


If we define $x_{1}(j)=1, y_{1}(j)=1, x_{3}(j)=-x(j)$ and $y_{3}(j)=-y(j)$, then

$$
U_{1}^{x}+U_{2}^{x} x_{2}(j)+U_{3}^{x}(-x(j))=0 \text { and } U_{1}^{y}+U_{2}^{y} y_{2}(j)+U_{3}^{y}(-y(j))=0 .
$$

From this definition and equation (5.5), we have that $U_{1}^{x} / U_{3}^{x}=c_{x}, U_{1}^{y} / U_{3}^{y}=c_{y}$, $U_{2}^{x} / U_{3}^{x}=s_{x}$ and $U_{2}^{y} / U_{3}^{y}=s_{y}$. Thus, a particular definition of the mapping functions $x_{k}(j)$ and $y_{k}(j)$ allow us to relate the projection of the dual projective elements directly with the parameters of a transformation. The relationship between the dual form of the projective space and the parameters of a transformation that define a shape in pattern matching is evident. In this case the dual form in the projective space can be interpreted as the location and scale parameters of a model shape $\boldsymbol{z}(j)=(u(j), v(j))$.

These ideas can be extended to consider other transformations useful in computer vision. For example, a linear transformation provides a good approximation of the projection of the silhouette of an object in an image when the object suffers a three-dimensional rigid motion. The dual definition of a primitive under this transformation can be obtained by replacing the transformation in equation (5.5) by a linear transformation. That is,

$$
\boldsymbol{x}(j)=\left[\begin{array}{ccc}
S_{1,1} & S_{1,2} & c_{x} \\
S_{2,1} & S_{2,2} & c_{y} \\
0 & 0 & 1
\end{array}\right]\left[\begin{array}{c}
u(j) \\
v(j) \\
1
\end{array}\right] .
$$

In this case the homogeneous form is given by four terms. That is,

$$
\begin{aligned}
U_{1}^{x} x_{1}(j)+U_{2}^{x} x_{2}(j)+U_{3}^{x} x_{3}(j)+U_{4}^{x} x_{4}(j) & =0, \\
U_{1}^{y} y_{1}(j)+U_{2}^{y} y_{2}(j)+U_{3}^{y} y_{3}(j)+U_{4}^{y} y_{4}(j) & =0 .
\end{aligned}
$$

By defining $x_{1}(j)=1, y_{1}(j)=1, x_{4}(j)=-x(j)$ and $y_{4}(j)=-y(j)$, then we have

$$
\left.\begin{array}{c}
U_{1}^{x}+U_{2}^{x} x_{2}(j)+U_{3}^{x} x_{3}(j)+U_{4}^{x}(-x(j))=0, \\
U_{1}^{y}+U_{2}^{y} y_{2}(j)+U_{3}^{y} y_{3}(j)+U_{4}^{y}(-y(j))=0 .
\end{array}\right\}
$$

By comparison of this definition and equation (5.7), we have that $x_{2}(j)=u(j)$, $x_{3}(j)=v(j), y_{2}(j)=u(j)$ and $y_{3}(j)=v(j)$. Hence the projection of the dual element is directly related to the transformation. That is,

$$
\begin{array}{lll}
U_{1}^{x} / U_{4}^{x}=c_{x}, & U_{2}^{x} / U_{4}^{x}=S_{1,1}, & U_{3}^{x} / U_{4}^{x}=S_{1,2}, \\
U_{1}^{y} / U_{4}^{y}=c_{y}, & U_{2}^{y} / U_{4}^{y}=S_{2,1}, & U_{3}^{y} / U_{4}^{y}=S_{2,2} .
\end{array}
$$

Thus, the dual element of the transformation of a shape under an affine transformation corresponds to a point in the projective dual space, and the projection of the dual element corresponds to the parameters of the transformation.

In general, the relationship between the parametrization of a form and the projection of the dual element, discussed in this section, is maintained for nonlinear transformations. We can consider the case of a projective transformation that can provide an accurate model of the formation of the silhouette of an object in an image. In this case equation (5.5) is replaced by

$$
\boldsymbol{x}(j)=\left[\begin{array}{ccc}
S_{1,1} & S_{1,2} & c_{x} \\
S_{2,1} & S_{2,2} & c_{y} \\
S_{3,1} & S_{3,2} & 1
\end{array}\right]\left[\begin{array}{c}
u(j) \\
v(j) \\
1
\end{array}\right],
$$


and the homogeneous form can be defined by six terms that define the functions

$$
\begin{array}{lll}
x_{1}(j)=1, & x_{2}(j)=u(j), & x_{3}(j)=v(j), \\
x_{4}(j)=-x(j) u(j), & x_{5}(j)=-x(j) v(j), & x_{6}(j)=-x(j), \\
y_{1}(j)=1, & y_{2}(j)=u(j), & y_{3}(j)=v(j), \\
y_{4}(j)=-y(j) u(j), & y_{5}(j)=-y(j) v(j), & y_{6}(j)=-y(j) .
\end{array}
$$

Thus, the projection of the dual element is related to the parameters of the transformation by

$$
\begin{array}{lllll}
\frac{U_{1}^{x}}{U_{6}^{x}}=c_{x}, & \frac{U_{2}^{x}}{U_{6}^{x}}=S_{1,1}, & \frac{U_{3}^{x}}{U_{6}^{x}}=S_{1,2}, & \frac{U_{4}^{x}}{U_{6}^{x}}=S_{3,1}, & \frac{U_{5}^{x}}{U_{6}^{x}}=S_{3,2}, \\
\frac{U_{1}^{y}}{U_{6}^{y}}=c_{y}, & \frac{U_{2}^{y}}{U_{6}^{y}}=S_{2,1}, & \frac{U_{3}^{y}}{U_{6}^{y}}=S_{y, 2}, & \frac{U_{4}^{y}}{U_{6}^{y}}=S_{3,1}, & \frac{U_{5}^{y}}{U_{6}^{y}}=S_{3,2} .
\end{array}
$$

In general, the parametrization in equation (5.2) defines a hyperplane and a point in the dual projective space, simultaneously. The definition of the HT in equations (2.7) and (5.4) computes the dual point. As shown in this section, each coordinate of the dual space can be related to the parameters of a transformation. In summary, the process of shape extraction defined in the HT for a general shape obtains the dual point defined according to the principle of duality.

For purposes of illustration, we can consider an example of the use of general forms in images. Figure $6 a, b$ shows two images of the same scene, taken from a different viewpoint. In this example, duality can be used to define a correspondence between the images by considering points represented by a parametric curve. In general, parametric curves have been used to represent consecutive pixels in images; however we can notice that the definition of $\boldsymbol{x}(j)$ in equation (5.1) can be used to represent any collection of points. In the example in figure 6, we have used corner points (Harris \& Stephens 1988) to determine the parameters of a linear transformation. First, we select a set of landmark points, shown in figure $6 c$, from the corners extracted from figure $6 a$. These points are selected by considering a high threshold value and they are used to define a parametric curve that can be matched against the corners extracted from figure $6 b$ and shown in figure $6 d$. The curve in figure $6 c$ suffices only to link the points: intermediate points are not necessarily related to image data. Due to the changes in the viewpoint the extracted corners are different; however, the transformation can be accurately determined by finding the dual point of the curve for the points in figure $6 d$.

We define the function $\boldsymbol{z}(j)=(u(j), v(j))$ such that it traces the curve that passes through the landmark points shown in figure $6 c$ when $j$ takes integer values between 1 and 9. The functions $u(j)$ and $v(j)$ were defined using a Fourier expansion (Aguado et al. 1998) as

$$
u(j)=\sum_{i=0}^{4} \rho_{i x} \cos \left(i j \frac{2}{9} \pi\right)+\rho_{i y} \sin \left(i j \frac{2}{9} \pi\right)
$$

for $\rho_{0}=(126,0), \rho_{1}=(30.6,-66.5), \rho_{2}=(3.23,-16.94), \rho_{3}=(24.77,5.96), \rho_{4}=$ $(31.20,-0.69)$ and

$$
v(j)=\sum_{i=0}^{4} \varphi_{i x} \cos \left(i j \frac{2}{9} \pi\right)+\varphi_{i y} \sin \left(i j \frac{2}{9} \pi\right)
$$



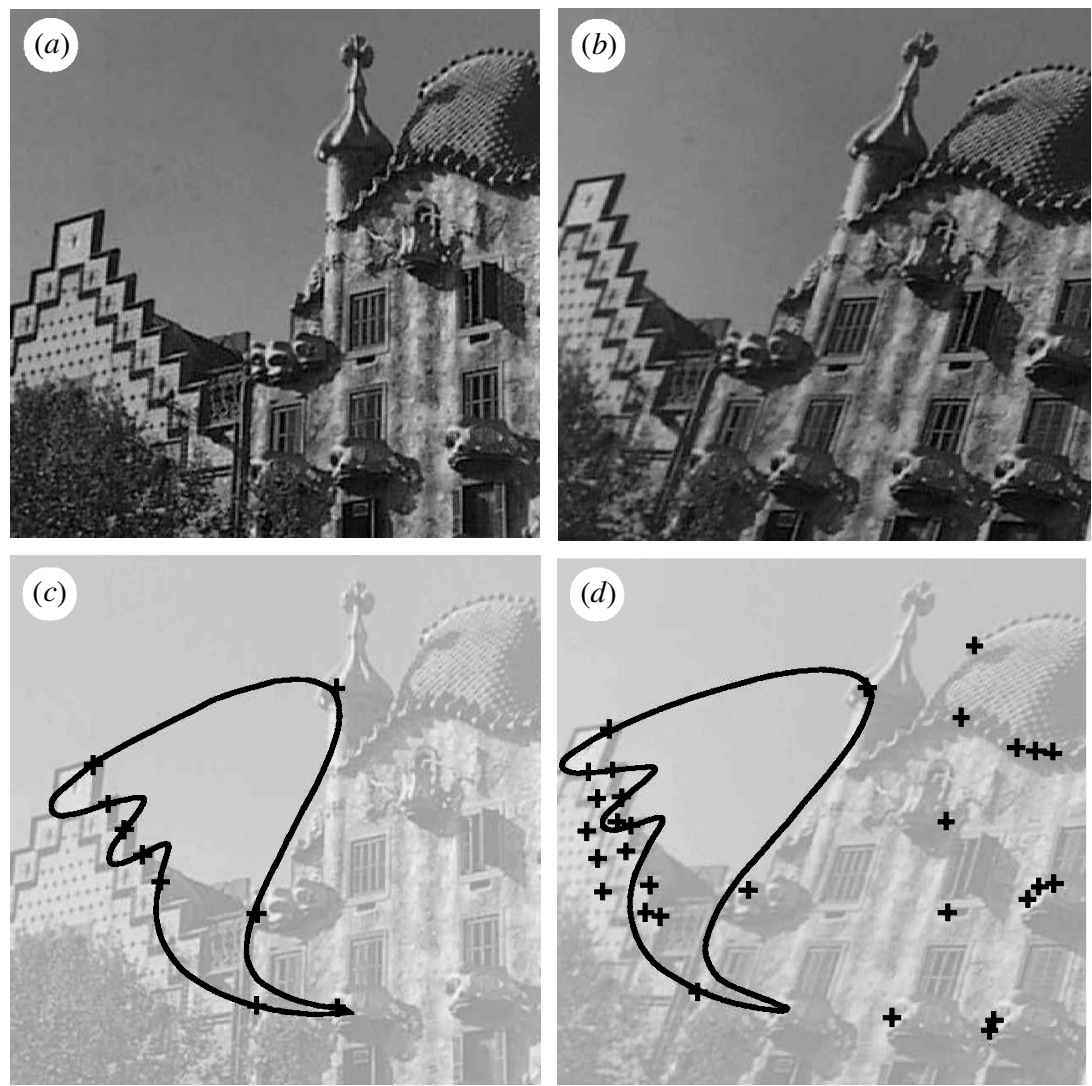

Figure 6. Example of forms under a geometrical transformation: $(a)$ and $(b)$, scene from different viewpoints; $(c)$ curve $\boldsymbol{z}(j) ;(d)$ curve defined by $\max \operatorname{HT}\left(\boldsymbol{U}_{i}\right)$ in equation (2.7).

for $\varphi_{0}=(149,0), \varphi_{1}=(38.7,67.98), \varphi_{2}=(45.95,6.72), \varphi_{3}=(13.22,-3.65)$, $\varphi_{4}=(17.34,6.86)$. If we consider $\boldsymbol{z}(j)$ in equation $(5.8)$, then the curve in figure $6 c$ has a dual point in the four-dimensional projective space, $\left[U_{1}^{x}, U_{2}^{x}, U_{3}^{x}, U_{4}^{x}\right]$ and $\left[U_{1}^{y}, U_{2}^{y}, U_{3}^{y}, U_{4}^{y}\right]$. Thus, each image point $(x(j), y(j))$ can be mapped into the points $\left(x_{1}, x_{2}, x_{3}, x_{4}\right)$ and $\left(y_{1}, y_{2}, y_{3}, y_{4}\right)$ according to the definitions in equation (5.8).

Based on the definition of the curve $\boldsymbol{z}(j)$ we can obtain the dual of the collection of corner points shown in figure $6 d$. Then, the dual defines the parameters in equation (5.7) and represents a linear transformation between figure $6 a$ and figure $6 b$. According to equation (2.7) the dual can be obtained by computing the value that satisfies the majority of equations simultaneously. In this example, equation (5.8) defines nine pairs of equations for each corner point. Thus, we have a system of 270 over-determined equations. In order to compute the maximum number of equations that can be satisfied simultaneously, we have taken groups of three equations to compute $\left[U_{1}^{x}, U_{2}^{x}, U_{3}^{x}, U_{4}^{x}\right]$ and $\left[U_{1}^{y}, U_{2}^{y}, U_{3}^{y}, U_{4}^{y}\right]$ in a similar way to the random consensus method (Fischler \& Bolles 1981). However, in order to reduce the number of combinations, we select groups of points based on a threshold value. In this way the first and second equations in the group only were chosen from the four points obtained by applying a high threshold in the corner detection process. Thus, only 
168 groups of points were considered. For each group we count how many of the equations satisfy the dual relationship in equation (5.2) and we keep the maximum value. Figure $6 d$ shows the curve obtained by the parameters in equation (2.3) for the dual point defined by the maximum value. This curve passes through six of the nine landmark points in figure $6 c$. This shows that the dual point defines the best-fit curve by the relationship between a point and a hyperplane in the projective space, illustrating how the new interpretation can be applied, with success, to real images. The points in figure $6 d$ through which the curve does not pass correspond to corner points not extracted from figure $6 a$.

\section{Conclusions and discussion}

We have considered the relationship between the HT and the principle of duality. The principle of duality was developed as a purely theoretical idea to explain theorems in dual projective spaces. The HT was developed as a pragmatic approach aimed at solving the fundamental problem of pattern matching. However, we have shown that there is a strong parallelism and convergence, not only in the basic and simple definitions but also in the development of ideas, concepts and properties. This relationship suggests a more general definition of the HT with a deep meaning in pure geometry rather than a simple mapping between coordinates. This definition is important for three main reasons. Firstly, it recognizes that the principle of duality has an important application in pattern matching. Secondly, it introduces the formalism of projective geometry to shape extraction and analysis. This increases our understanding of the dual nature of pattern matching, thus techniques can benefit from previous results obtained in projective geometry. Finally, the principle of duality provides a general definition, wherein the dual form of lines, curves and hyperplanes has a dual interpretation in pattern matching. We have discussed the interpretation of lines and points and we have generalized the ideas to represent hyperplanes and general shapes represented by parametric curves.

In order to show the relationship between the HT and the principle of duality, we have related the dual equations of geometry to the definition of the HT. Our development is extended to include shapes composed of simple primitives, conics and general parametric forms. From this development we draw four main conclusions. Firstly, the HT can be interpreted as an application of the principle of duality of geometry. Extended forms and properties of the HT have a corresponding development in the dual projective space. Secondly, there is an equivalence between the Hough space and the projective space, since the Hough space corresponds to a projection of the projective space. The projection of the projective space into the Euclidean space defines a parametric form of a shape. The properties and topology of the primal and dual spaces are determined by the relationships in the dual space defined by projective geometry. When the projected form is obtained by considering an orthogonal definition of a general curve and a transformation, then the parameters of the transformation are directly related to each coordinate of the dual space. This relationship proves the equivalence between the Hough space and the dual projective space. Thirdly, the principle of duality defines an over-determined system. The HT implicitly solves for this system by computing the solution that satisfies the largest number of equations simultaneously, thus maxima define dual elements. Accordingly, the HT defines a computational approach to apply the prin- 
ciple of duality. However, the definition and mathematical properties of the Hough space and the dual projective space are equivalent. Finally, the HT and the principle of duality define a one-to-one linear mapping. Dual elements of a figure represent dual elements of a shape. Points, lines and hyperplanes define a linear mapping in homogeneous coordinates.

The principle of duality constitutes an important concept for understanding geometric relationships in the projective space. The use of duality in the HT can provide a natural definition from which theorems and properties can be demonstrated. In an integrated approach, the geometric relationships in the projective space and the computational definition of the HT provide two complementary areas which are both necessary to solve demanding pattern matching applications.

We are very grateful for the comments and suggestions of the (anonymous) referees.

\section{References}

Aguado, A. S., Nixon, M. S. \& Montiel, M. E. 1998 Parameterising arbitrary shapes via Fourier descriptors for evidence-gathering. CVIU Image Understanding 69, 202-221.

Baker, H. F. 1971 An introduction to planar geometry with many examples. New York: Chelsea.

Ballard, D. H 1981 Generalizing the Hough transform to detect arbitrary shapes. Pattern Recognition 13, 111-122.

Buchanan, T. 1988 The twisted cubic and camera calibration. Computer Vision Graphics Image Processing 42, 130-132.

Canny, J. F. 1983 Finding edges and lines in images. Report AI-TR-720, MIT Artificial Intelligence Laboratory.

Deans, S. R. 1981 Hough transform from the radon transform. IEEE Trans. Pattern Analysis Machine Intell. 13, 185-188.

Duda, R. O. \& Hart, P. E. 1972 Use of the Hough transform to detect lines and curves in pictures. Commun. ACM 13, 11-15.

Duda, R. O. \& Hart, P. E. 1973 Pattern classification and scene analysis. Wiley.

Eckhardt, U. \& Maderlechner, G. 1990 A general approach for parameterising the Hough transform. IEEE 10th Int. Conf. on Pattern Recognition, pp. 623-630. IEEE.

Fishback, W. T. 1969 Projective and Euclidean geometry. Wiley.

Fischler, M. A. \& Bolles, R. C. 1981 Random sample consensus: a paradigm for model fitting with applications to image analysis and automated cartography. Graphics Image Processing 24, 381-395.

Harris, C. \& Stephens, M. 1988 A combined corner and edge detector. Proc. Alvey Vision Conf., pp. 147-151. University of Manchester.

Hough, P. V. 1962 Method and means for recognising complex patterns. US Patent no. 3969654.

Illingworth, J. \& Kittler, J. 1988 A survey of the Hough transform. Computer Vision Graphics Image Processing 48, 87-116.

Julesz, B. 1971 Foundations of cyclopean perception. The University of Chicago Press.

Leavers, V. F. 1993 Which Hough transform? CVGIP: Image Understanding 58, 250-264.

Marr, D. \& Nishihara, K 1978 Representation and recognition of the spatial organization of three-dimensional shapes. Proc. R. Soc. Lond. B 200, 269-294.

Mundy, J. L. \& Zisserman, A. 1992 Geometric invariance in computer vision. MIT Press.

Pedoe, D. 1963 International series of monographs in pure and applied mathematics. Pergamon.

Pentland, A. P. 1986 Perceptual organization and the representation of natural form. Artificial Intell. 28, 293-331.

Plücker, J. 1865 On a new geometry of space. Phil. Trans. R. Soc. Lond. 155, 725-791.

Proc. R. Soc. Lond. A (2000) 
Princen, J., Illingworth, J. \& Kittler, J. 1992 A formal definition of the Hough transform properties and relationships. J. Math. Imaging Vision 1, 153-168.

Rosenfeld, A. 1969 Picture processing by computer. Academic.

Rosenfeld, A. \& Weiss, I. 1995 A convex polygon is determined by its Hough transform. Pattern Recognition Lett. 16, 305-306

Roth, G. \& Levine, M. D. 1993 Extracting natural scales using Fourier descriptors. Pattern Recognition 26, 1383-1893.

Sklansky, J. 1978 On the Hough technique for curve detection. IEEE Trans. Computers 27, 923-926.

Stahs, T. G. \& Wahl, F. M. 1989 Polyhedral object recognition by Hough space analysis. Mathematical research, vol. 51, pp. 165-172. Berlin: Akademie.

Stockman, G. C. \& Agwarwala, A. K. 1977 Equivalence of Hough curve detection to template matching. Commun. ACM 20, 820-822.

Tuller, A. 1967 A modern introduction to geometries. The university series in undergraduate mathematics. Princeton, NJ: Van Nostrand.

Veblen, O. \& Young, J. W. 1910 Projective geometry, vol. 1. London: Ginn \& Company.

Wahl, F. M. 1989 Deriving features from Hough space for object recognition and configuration estimation. In From pixels to features (ed. J. C. Simon), pp. 141-152. Elsevier. 